\title{
Diphtheria toxoid dissolving microneedle vaccination: Adjuvant screening and effect of repeated-fractional dose administration
}

\author{
M. Leone ${ }^{a}$, S. Romeijn ${ }^{a}$, G. Du ${ }^{a}$, S.E. Le Dévédec ${ }^{\mathrm{b}}$, H. Vrieling ${ }^{\mathrm{a}, \mathrm{d}}$, C. O'Mahony $^{\mathrm{c}}$, J.A. Bouwstra ${ }^{\mathrm{a}, *, 1}$, \\ G. Kersten ${ }^{\mathrm{a}, \mathrm{d}, 1}$ \\ ${ }^{a}$ Division of BioTherapeutics, Leiden Academic Centre for Drug Research, Leiden University, the Netherlands \\ ${ }^{\mathrm{b}}$ Division of Drug Discovery and Safety, Leiden Academic Centre for Drug Research, Leiden University, the Netherlands \\ ${ }^{\mathrm{c}}$ Tyndall National Institute, University College Cork, Cork, Ireland \\ ${ }^{\mathrm{d}}$ Institute for Translational Vaccinology, Bilthoven, the Netherlands
}

\section{A R T I C L E I N F O}

\section{Keywords:}

Dissolving microneedles

Diphtheria toxoid

Intradermal immunization

Microneedles

Aluminum-based adjuvants

Repeated-fractional vaccine delivery

\begin{abstract}
A B S T R A C T
In this study the effect of repeated-fractional intradermal administration of diphtheria toxoid (DT) compared to a single administration in the presence or absence of adjuvants formulated in dissolving microneedles (dMNs) was investigated. Based on an adjuvant screening with a hollow microneedle (hMN) system, poly(I:C) and gibbsite, a nanoparticulate aluminum salt, were selected for further studies: they were co-encapsulated with DT in dMNs with either a full or fractional DT-adjuvant dose. Sharp dMNs were prepared regardless the composition and were capable to penetrate the skin, dissolve within $20 \mathrm{~min}$ and deposit the intended antigen-adjuvant dose, which remained in the skin for at least $5 \mathrm{~h}$. Dermal immunization with hMN in repeated-fractional dosing (RFrD) resulted in a higher immune response than a single-full dose (SFD) administration. Vaccination by dMNs led overall to higher responses than hMN but did not show an enhanced response after RFrD compared to a SFD administration. Co-encapsulation of the adjuvant in dMNs did not increase the immune response further. Immunization by dMNs without adjuvant gave a comparable response to subcutaneously injected DT-AlPO in a 15 times higher dose of DT, as well as subcutaneous injected DT-poly(I:C) in a similar DT dose. Summarizing, adjuvant-free dMNs showed to be a promising delivery tool for vaccination performed in SFD administration.
\end{abstract}

\section{Introduction}

Vaccination has led to the control of devastating diseases such as smallpox, poliomyelitis, measles and hepatitis (Du et al., 2018; Jiang et al., 2017; Peek et al., 2008). Most vaccines are injected intramuscularly or subcutaneously. However, classical injection can cause pain, distress, needle-stick injuries and requires trained personnel (Leone et al., 2017). To overcome problems related to the hypodermic needles, less invasive technologies have been developed such as microneedles (Larraneta et al. 2016; Leone et al., 2017). Microneedles are structures up to $1 \mathrm{~mm}$ in length capable to penetrate the stratum corneum, the major skin barrier, in a pain-free way (Leone et al., 2017; van der Maaden et al., 2012), thereby delivering the antigen into the skin, a very immune competent organ populated with many antigen presenting cells. This may lead to antigen dose-sparing (Leone et al., 2017; Li et al., 2011) compared to the conventional routes of administration.
Dissolving microneedles (dMNs) are a microneedle type that dissolve in the skin upon insertion thereby releasing the encapsulated vaccine (Leone et al., 2017). Their dissolution in the skin allows to avoid sharp needle waste left behind after use and thus infections due to the needle re-use or needle-stick injuries are not possible (Kim et al., 2012). Furthermore, for vaccine in the solid state it may be possible to circumvent the need for a cold-chain to keep the antigen stable during storage and shipping (Gill and Prausnitz, 2007).

Vaccines consist of attenuated organisms, inactivated pathogens and toxins or subunit antigens. While attenuated vaccines may revert to the virulent form, inactivated and subunit vaccines are safer but generally less immunogenic (Peek et al., 2008; Reed et al., 2013). Thus, to potentiate the immune response using safer vaccines, together with the optimal administration route, adjuvants can be used, aiming for increased immunogenicity or antigen dose-sparing (Leone et al. 2017; Reed et al., 2013; Kumru et al., 2014).

\footnotetext{
* Corresponding author at: Cluster BioTherapeutics, Leiden Academic Centre for Drug Research, Leiden University, Einsteinweg 55, P.O. Box 9502, 2300 RA Leiden, the Netherlands.

E-mail address: bouwstra@lacdr.leidenuniv.nl (J.A. Bouwstra).

${ }^{1}$ Contributed equally.
} 
However, adjuvants can have drawbacks such as adverse effects and they may affect vaccine stability (Kumru et al., 2014). A previous study revealed that repeated administration of fractional doses of inactivated polio vaccine by means of a hollow microneedle (hMN) can lead to superior IgG responses without the use of adjuvants (Schipper et al., 2016a).

In this study, we examined whether the immunogenicity of diphtheria toxoid (DT) can be influenced by repeated dermal administration in comparison with a single dose without or with the addition of adjuvants. In a previous study the effect of repeated antigen dosing was assessed by using hMN (Schipper et al., 2016a). In the present research, it was investigated whether repeated dosing by using dMNs and hMN had a similar effect on the immune response. To select the optimal adjuvant, the vaccination was performed by using a hMN intradermally in mice. This allowed to avoid time consuming dMN fabrication for all adjuvants and to screen a quite wide adjuvant set and to perform a relatively fast selection of them to encapsulate in the dMNs. Based on these studies, two adjuvants were selected for a follow-up study in which intradermal administration of DT and the adjuvant was performed in a single-full dose or in repeated-fractional dosing using either a hMN or dMNs.

\section{Materials and methods}

\subsection{Materials}

Hyaluronan (sodium hyaluronate, HA, average Mw was $150 \mathrm{kDa}$ ) was purchased from Lifecore Biomedical (Chaska, MN, USA). Diphtheria toxoid (DT) $(12.25 \mathrm{mg} / \mathrm{mL}$ in Phosphate buffered saline (PBS) $\mathrm{pH} 7.4)$ and diphtheria toxin $(0.001 \mathrm{Lf} / \mathrm{ml})$ were kindly provided by Intravacc (Bilthoven, The Netherlands). CpG ODN 1826 was purchased from Oligo Factory (Holliston, MA). Aluminum phosphate $\left(\mathrm{AlPO}_{4}\right)$ was purchased from Brenntag (Ballerup, Denmark). Fetal bovine serum (FBS) and cholera toxin (vibrio cholera) were obtained from Sigma-Aldrich (Zwijndrecht, The Netherlands). Glucose solution, LGlutamine $(200 \mathrm{mM})$, penicillin-streptomycin $(10000 \mathrm{U} / \mathrm{ml})$, and sodium bicarbonate were obtained from (Thermo-Fisher Scientific, Waltham, MA). Polyinosinic-polycytidylic acid (poly(I:C)) (low molecular weight) was purchased from Invivogen (Toulouse, France). Sterile phosphate buffered saline (PBS, $163.9 \mathrm{mM} \mathrm{Na}^{+}, 140.3 \mathrm{mM} \mathrm{Cl}^{-}$, $8.7 \mathrm{mM} \mathrm{HPO}_{4}{ }^{2-}, 1.8 \mathrm{mM} \mathrm{H}_{2} \mathrm{PO}_{4}^{-}$, $\mathrm{pH} 7.4$ ) was ordered from Braun (Oss, The Netherlands). $10 \mathrm{mM}$ phosphate buffer (PB, $7.7 \mathrm{mM}$ $\mathrm{Na}_{2} \mathrm{HPO}_{4}, 2.3 \mathrm{mM} \mathrm{NaH} \mathrm{PO}_{4}, \mathrm{pH}$ 7.4) was prepared in the laboratory. All the chemicals used were of analytical grade and distilled water (18 $\mathrm{M} \Omega / \mathrm{cm}$, Millipore Co.) was used for the preparation of all solutions.

\subsection{Synthesis of boehmite and gibbsite}

Nanoparticulate aluminum salts boehmite and gibbsite were synthesized as described previously (Buining et al., 1991; Wierenga et al., 1998). Aluminium-iso-propoxide ( $80 \mathrm{mM})$ and aluminum-sec-butoxide ( $80 \mathrm{mM})$ were mixed in $\mathrm{HCl}(90 \mathrm{mM})$ and stirred for 10 days. The solution was hydrothermally treated at $150{ }^{\circ} \mathrm{C}$ (boehmite) or $85{ }^{\circ} \mathrm{C}$ (gibbsite) for $36 \mathrm{~h}$. The suspensions were dialyzed against water, autoclaved and stored at room temperature.

\subsection{Preparation of formulations for hollow microneedle injections}

In order to select the most promising adjuvants, various DT-adjuvant formulations were tested (Table 1) using a hMN injection system. DT was mixed in a concentration of $36 \mu \mathrm{g} / \mathrm{ml}$ with i) CpG ODN (36 $\mu \mathrm{g} / \mathrm{ml})$ or poly(I:C) $(36 \mu \mathrm{g} / \mathrm{ml})$ or cholera toxin $(100 \mu \mathrm{g} / \mathrm{ml})$ in PBS (pH 7.4), ii) the aluminum-based nanoparticles (alumNPs) gibbsite or boehmite $\left(36 \mu \mathrm{g} / \mathrm{ml} \mathrm{Al}^{3+}\right.$ or $\left.360 \mu \mathrm{g} / \mathrm{ml} \mathrm{Al}^{3+}\right)$ in a sucrose $(250 \mathrm{mM})$ containing histidine buffer (50 mM, pH 7.5). For the positive control, DT in a concentration of $500 \mu \mathrm{g} / \mathrm{ml}$ was mixed with $\mathrm{AlPO}_{4}$ in a concentration of $15 \mathrm{mg} / \mathrm{ml}\left(\mathrm{DT}-\mathrm{AlPO}_{4}\right.$ ) in PBS (pH 7.4). AlumNPs and DT-AlPO ${ }_{4}$ were incubated under continuous stirring at room temperature for $3 \mathrm{~h}$ to allow the DT adsorption on alumNPs or $\mathrm{AlPO}_{4}$.

\subsection{DT adsorption on alumNPs and $\mathrm{AlPO}_{4}$}

To determine the adsorption of free DT to alumNPs or $\mathrm{AlPO}_{4}$, after the adsorption procedure the samples were centrifuged for $60 \mathrm{~min}$ at $35,000 \times \mathrm{g}$ at $4{ }^{\circ} \mathrm{C}$ in an Avanti J-20 XP centrifuge (Beckman Coulter). The DT in the supernatant was quantified by measuring the intrinsic fluorescence intensity of DT ( $\left.\lambda_{\mathrm{ex}} 280 \mathrm{~nm} / \lambda_{\mathrm{em}} 320 \mathrm{~nm}\right)$. The adsorption efficiency of DT was calculated according to the following equation:

Adsorption efficieny $\%=\left(1-\frac{M_{D T} \text { in supernatant }}{M_{D T} \text { total }}\right) \times 100 \%$

where $M_{D T}$ in supernatant represents the mass of DT in supernatant after centrifugation, and $M_{D T}$ total is the total mass of DT used.

\subsection{Particles size and zeta potential determination}

For DT-alumNPs (DT in concentration of $36 \mu \mathrm{g} / \mathrm{ml}$, alumNPs in concentrations of $36 \mu \mathrm{g} / \mathrm{ml} \mathrm{Al}^{3+}$ or $360 \mu \mathrm{g} / \mathrm{ml} \mathrm{Al}^{3+}$ ) the particle size, polydispersity index (PDI) and zeta potential were determined by dynamic light scattering (DLS) and laser doppler velocimetry (Nano $\mathrm{ZS}^{\circledR}$ zetasizer, Malvern Instruments, Worcestershire, U.K.). To resemble the conditions for hMN injection and dMN arrays fabrication, samples were respectively prepared in $50 \mathrm{mM}$ histidine $(\mathrm{pH} 7.5)$ or in $10 \mathrm{mM} \mathrm{PB} \mathrm{(pH}$ 7.4).

\subsection{Labeling of diphtheria, hyaluronan and alumNPs}

\subsubsection{Labeling for confocal microscopy}

DT was labelled with Alexa Fluor 647 ${ }^{\circledR}$ dye (AF647) (Life Technologies, Eugene, OR, USA) ( $\lambda$ ex $651 \mathrm{~nm}, \lambda$ em $672 \mathrm{~nm}$ ) (DTAF647) according to the manufacturer's instructions. Hyaluronan was labelled with fluoresceinamine (FAM) (Sigma-Aldrich, St. Louis, MO, USA) (isomer I, $\lambda$ ex $496 \mathrm{~nm}$, $\lambda$ em $520 \mathrm{~nm}$ ) (HA-FAM) following the method described by de Belder and Wik (1975). Gibbsite was labelled with lumogallion (Gib-LMG) (TCI Europe N.V., Antwerp, Belgium) ( $\lambda$ ex $493 \mathrm{~nm}$, $\lambda$ em $600 \mathrm{~nm}$ ) following the method described by Mile et al. (2015).

\subsubsection{Labeling for infrared detection}

DT deposition in ex vivo-mouse and -human skin was quantified by using DT labelled with IRDye 800CW (LI-COR, Lincoln, Nebraska USA) ( $\lambda$ ex $774 \mathrm{~nm}, \lambda$ em $789 \mathrm{~nm}$ ). DT labelling was performed according to the manufacturer's instructions.

\subsection{Fabrication of dissolving microneedle arrays}

dMN arrays ( $4 \times 4$ needles) were prepared as previously described (Leone et al., 2019, 2018b). Briefly, 10\% (w/v) HA was dissolved in PB (10 mM, pH 7.4) and stored overnight. The next day, $0.3 \%(\mathrm{w} / \mathrm{v})$ DT for the full dose dMNs or $0.1 \%(\mathrm{w} / \mathrm{v})$ DT for the fractional dose dMNs was added to the HA solution. For adjuvanted dMNs, the adjuvant in a weight ratio 1:1 with DT was added.

A polydimethylsiloxane mold (PDMS, Sylgard 184, Dow Corning, Midland, MI, USA) consisting of single-array wells was made using a master template presenting solid silicon MN arrays (Leone et al., 2019). The PDMS mold was used to pour the HA-DT solution in each well. After several vacuum cycles and centrifugation steps, the dMN arrays were dried at $37{ }^{\circ} \mathrm{C}$ overnight. The next day, an antigen-free backplate was produced by pouring a mixture of vinylpolysiloxane base and catalyst (1:1 ratio) (Elite Double 32a Normal, Zhermack Group, Badia Polesine, Italy). Subsequently a two-component glue solution (Bison 
Table 1

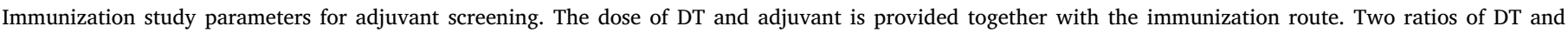
alumNPs were used.

\begin{tabular}{|c|c|c|c|c|c|c|c|c|c|c|}
\hline \multirow{3}{*}{$\begin{array}{l}\text { Immunization route } \\
\text { Group name }^{*}\end{array}$} & \multicolumn{9}{|c|}{ Intradermal by hollow microneedle } & \multirow{3}{*}{$\begin{array}{l}\text { Subcutaneous } \\
\text { DT- } \mathrm{AlPO}_{4} \mathrm{sc}\end{array}$} \\
\hline & \multirow[t]{2}{*}{ DT } & \multirow[t]{2}{*}{ DT-CT } & \multirow[t]{2}{*}{ DT-PI } & \multirow[t]{2}{*}{ DT-CpG ODN } & \multicolumn{2}{|l|}{ DT-Gib } & \multicolumn{2}{|l|}{ DT-Boe } & \multirow[t]{2}{*}{ PBS } & \\
\hline & & & & & $1: 1$ & $1: 10$ & $1: 1$ & $1: 10$ & & \\
\hline DT dose $(\mu \mathrm{g})$ & 0.36 & & & & & & & & - & 5 \\
\hline Adjuvant dose $(\mu \mathrm{g})$ & - & 1 & 0.36 & & $0.36^{* *)}$ & $3.6^{* *}$ & $0.36^{* * *}$ & $\left.3.6^{* *}\right)$ & - & 150 \\
\hline
\end{tabular}

*) DT: diphtheria toxoid; CT: cholera toxin; PI: poly(I:C); Gib: gibbsite; Boe: boehmite; AlPO 4 : aluminum phosphate.

**) $\mathrm{Al}^{3+}$ concentration.

International B.V., Goes, The Netherlands) was poured onto each array and left curing. Finally, the arrays were removed from the PDMS mold, cut into individual arrays and stored at room temperature in a desiccator until use.

To perform confocal imaging, in preparing the dMN arrays $100 \%$ of the DT and gibbsite amount and $4.5 \%$ of the hyaluronan amount were replaced with their labelled counterparts (DT-AF647, Gib-LMG and HAFAM respectively). To perform near-infrared imaging of DT in the skin, $36 \%$ of the full dose of DT and $100 \%$ of the fractional dose of DT was labelled (DT-IR800).

\subsection{Human skin}

Human abdomen skin was obtained from a local hospital within $24 \mathrm{~h}$ after cosmetic surgery. After removal of the fat excess with a scalpel, the skin was placed in the $-80^{\circ} \mathrm{C}$ freezer until use. Before use, the skin was thawed in a petri dish containing a wet tissue at $37{ }^{\circ} \mathrm{C}$ for $1 \mathrm{~h}$ and stretched with pins on parafilm-covered styrofoam. The skin was cleaned with distilled water and $70 \%$ ethanol before the start of the experiment.

Fresh ex vivo human skin was used within $24 \mathrm{~h}$ after cosmetic surgery. After manual removal of the fat excess, the skin was cleaned with Milli-Q and 70\% ethanol and stretched with pins on parafilm-covered Styrofoam to be used.

\subsection{Penetration of microneedles in ex vivo human skin}

dMN arrays $(n=3)$ were applied onto the skin by impact velocity, as described elsewhere (Leone et al., 2018b), by using an impact insertion applicator with a constant velocity of $0.40 \mathrm{~m} / \mathrm{s}$ (Leiden University - applicator with uPRAX controller version 0.3 ) and kept in the skin during $18 \mathrm{sec}$. Penetration efficiency (PE) was determined by trypan blue treatment of pierced skin, as previously described (van der Maaden et al., 2014a). After removal of the stratum corneum by stripping the blue spots were visualized using a light microscope. The penetration efficiency per array was calculated as follows (Eq. (2)), in which 16 is the number of microneedles per array:

Penetration efficiency $=\frac{\text { Number of blue spots }}{16} \times 100$

\subsection{Dissolution of microneedles in ex vivo human skin}

dMNs arrays $(n=3)$ were applied on the skin as previously described (Section 2.9) and were kept by the applicator for $20 \mathrm{~min}$ in the skin. The microneedle length before and after dissolution was determined with a light microscope (Axioskop and Stemi 2000-C, Carl Zeiss Microscopy GmbH, Göttingen, Germany) equipped with a digital camera (Axiocam ICc 5, Carl Zeiss). The images were analysed by ZEN 2012 blue edition software (Carl Zeiss Microscopy GmbH). The dissolved $\mathrm{MN}$ volumes were calculated as reported previously (Leone et al., 2019).

\subsection{Quantification of diphtheria delivered in ex vivo mouse and human} skin

Full dose dMN arrays ( $n=3$ per skin type) and fractional dose dMN arrays ( $\mathrm{n}=3$ per skin type) were inserted into mouse or human skin $e x$ vivo and the dMNs remained in the skin for $20 \mathrm{~min}$. After dMN array removal, the near-infrared fluorescence of the delivered DT-IR800 was measured in a Perkin-Elmer IVIS Lumina Series III in vivo imaging system (Waltham, MA, USA), by using a ICG bkg excitation filter and an ICG emission filter and acquisition time $4 \mathrm{~s}$, F-stop 2, binning 4 and field of view of $12.5 \mathrm{~cm}$. Perkin-Elmer Living Image software version 4.3.1.0 was used for image acquisition and analysis. Fluorescence data were processed using region of interest (ROI) analysis with background subtraction consisting of a control region of the skin.

A calibration curve was generated in mouse and human skin by intradermal microinjections of DT-IR800 of 62.5-1000 ng with an in house fabricated hMN injection system with UPRAX controller version 0.3 (Leiden University) as reported elsewhere (Schipper et al., 2016a, 2016b; van der Maaden et al., 2014b).

\subsection{Confocal laser scanning microscopy}

Confocal laser scanning microscopy (CLSM) was performed with a Nikon TE-2000-e inverted microscope equipped with a C1 confocal unit. Nikon Plan Apo $10 \times$ and $4 \times$ objectives (with a numerical aperture of 0.20 and 0.45 and working distance of 15.7 and 4 respectively) were used respectively for microneedle and skin visualization. Nikon NIS Elements version 4.20.00 64-bit software was used for acquisition and analysis of scans.

For dMN visualization, fluoresceinamine (FAM) and lumogallion (LMG) were excited at $488 \mathrm{~nm}$ and Alexa Fluor 647 at $633 \mathrm{~nm}$. The xy resolution was $1.55 \mu \mathrm{m} /$ pixel.

For antigen and nanoparticle localization in the skin, fluorescently labelled dMNs were inserted into ex vivo fresh human skin for 20 min. After removal of the remaining dMN array, time-lapse microscopy using CLSM as described above was performed with the skin in order to visualize hyaluronan, gibbsite or DT respectively. Each $30 \mathrm{~min}$, sequentially xy scans (xy resolution of $6.21 \mu \mathrm{m} /$ pixel) were taken with a spatial resolution of $10 \mu \mathrm{m}$ in z-direction (z-axis of $0.7 \mathrm{~mm}$ ) over a time period of $5 \mathrm{~h}$.

\subsection{Immunization studies}

Immunization studies were performed using female BALB/c mice (H2d), 8-11 weeks old (Charles River, Maastricht, The Netherlands). The studies were approved by the ethical committee on animal experiments of Leiden University (License number 14241). The mice were randomly assigned to groups of 8 .

Immunizations were given at day 1 (prime immunization), day 22 (boost immunization) and day 43 (2nd boost immunization). Before each intradermal immunization, the mice were shaved on the left flank (approximately $4 \mathrm{~cm}^{2}$ ). A blood sample was collected, serum was isolated and stored at $-80{ }^{\circ} \mathrm{C}$. Prior to vaccination, mice were anesthetized by intraperitoneal injection of $150 \mathrm{mg} / \mathrm{kg}$ ketamine and $10 \mathrm{mg} / \mathrm{kg}$ 
xylazine. At day 63, all mice were sacrificed and serum was collected.

\subsubsection{Part I: Adjuvant screening}

The effect of adjuvants on the immunogenicity of dermally injected DT was assessed using hMN injection (Table 1). The inner diameter of the hMN was approximately of $150 \mu \mathrm{m}$ and the length of the microneedle tip of approximately $120 \mu \mathrm{m}$. Injected volume was $10 \mu \mathrm{l}$ at a controlled depth of $120 \mu \mathrm{m}$ by using a specifically designed hMN injection system with uPRAX controller version 0.3 (Leiden University) (van der Maaden et al., 2014b). Negative and positive controls included respectively intradermal injection of PBS by hMN and $100 \mu \mathrm{l}$ subcutaneous injection of $5 \mu \mathrm{g}$ DT and $150 \mu \mathrm{g} \mathrm{AlPO}_{4}$ with a conventional $26 \mathrm{G}$ needle.

\subsubsection{Part II: single-full dose vs repeated-fractional doses}

The effects of DT administration, with and without adjuvant, in repeated-fractional doses (RFrD) were investigated and compared to a single-full dose (SFD) injection (100\% dose). The RFrD consisted of administration, in 3 consecutive days, of $1 / 3$ rd of the SFD of DT(-adjuvant) $(3 \times 33 \%$ doses $)$. Intradermal vaccination in mice was performed using hMN (10 $\mu \mathrm{l}$ at a depth of $120 \mu \mathrm{m}$ ) and dMNs. Details of the formulations are reported in Table 2. PBS and DT- $\mathrm{AlPO}_{4}$ groups were used as negative and positive control, respectively. An additional positive control of subcutaneous injection of $100 \mu \mathrm{l}$ of $0.36 \mu \mathrm{g}$ DT and $0.36 \mu \mathrm{g}$ poly(I:C) was included.

Table 2. Immunization study parameters for administration kinetics investigation. The administration is in SFD (100\%) or in $3 \mathrm{RFrD}$ $(3 \times 33 \%)$.

*) the DT and adjuvant dose are equal except for the subcutaneous injection with $\mathrm{AlPO}_{4}$, in which $5 \mu \mathrm{g}$ of DT and $150 \mu \mathrm{g} \mathrm{AlPO}$ has been added in the formulation; ${ }^{* *}$ application of an empty dMN array or injection of PBS on 2 consecutive days and DT administration in SFD on day 3. Abbreviations are DT: diphtheria toxoid; Gib: gibbsite; PI: poly (I:C); $\mathrm{AlPO}_{4}$ : aluminum phosphate; E: empty dMN array.

Table 2

Immunization study parameters for administration kinetics investigation. The administration is in SFD (100\%) or in $3 \operatorname{RFrD}(3 \times 33 \%)$.

\begin{tabular}{lll}
\hline Formulation & $\begin{array}{l}\text { Formulation dose administration } \\
\text { schedule }\end{array}$ & $\begin{array}{l}\text { DT dose per array/ } \\
\text { injection }(\mu \mathrm{g})\end{array}$ \\
\hline \multicolumn{2}{l}{ Intradermal administration: dissolving microneedles } \\
DT & SFD & 0.36 \\
DT-Gib & & \\
DT-PI & & \\
E/E/DT & \\
DT & RFrD & 0.12 \\
DT-Gib & & \\
DT-PI & & \\
Intradermal injection: hollow microneedles & \\
DT & SFD & 0.36 \\
PBS/PBS/DT & \\
DT & RFrD & 0.12 \\
PBS & - & - \\
Subcutaneous injection (conventional 26G needle) & \\
DT-PI & SFD & 0.36 \\
DT-AlPO & SFD & 5 \\
\hline
\end{tabular}

*) the DT and adjuvant dose are equal except for the subcutaneous injection with $\mathrm{AlPO}_{4}$, in which $5 \mu \mathrm{g}$ of DT and $150 \mu \mathrm{g} \mathrm{AlPO}_{4}$ has been added in the formulation;

$* *$ ) application of an empty dMN array or injection of PBS on 2 consecutive days and DT administration in SFD on day 3. Abbreviations are DT: diphtheria toxoid; Gib: gibbsite; PI: poly(I:C); $\mathrm{AlPO}_{4}$ : aluminum phosphate; E: empty dMN array.

\subsection{Determination of DT-specific serum IgG titers and diphtheria toxin- neutralizing antibody titers}

DT-specific total IgG, IgG1 and IgG2a titers in serum were determined by ELISA as described previously (Schipper et al., 2017). Plates were coated with $140 \mathrm{ng}$ DT per well and incubated overnight at $4{ }^{\circ} \mathrm{C}$. After blocking with BSA (Sigma-Aldrich, Zwijndrecht, The Netherlands), sera samples were added in a three-fold serial dilution and the plates were incubated at $37{ }^{\circ} \mathrm{C}$ for $2 \mathrm{~h}$. Detection of antibodies was performed with horseradish peroxidase-conjugated goat-anti-mouse IgG, IgG1 or IgG2a (Southern Biotech, Birmingham, AL) (1:5000 dilution) using 1-stepTM ultra 3,3',5,5'-tetramethylbenzidine (TMB) (Thermo-Fisher Scientific, Waltham, MA) as substrate. The reaction was stopped with $2 \mathrm{M}$ sulfuric acid (JT Baker, Deventer, The Netherlands). Absorbance was measured at $450 \mathrm{~nm}$. Antibody titers were expressed as the $\log 10$ value of the serum dilution at the mid-point of the S-shaped absorbance-dilution curve.

Toxin neutralizing capacity of antisera was measured in a Vero cell assay (Ding et al., 2009). After complement inactivation by heating at $56{ }^{\circ} \mathrm{C}$ for $45 \mathrm{~min}$, appropriate two-fold serial dilutions of serum samples were prepared in M199 medium (Sigma-Aldrich, Zwijndrecht, The Netherlands) (supplemented with 5\% FBS (Sigma-Aldrich, Zwijndrecht, The Netherlands), $1 \mathrm{~g} / 1$ glucose, $1.6 \mathrm{mM}$ L-glutamine, $1.7 \mathrm{~g} / 1$ sodium bicarbonate and $100 \mathrm{U} / \mathrm{ml}$ penicillin-streptomycin) and were applied to 96 -well plates. Subsequently, $50 \mu \mathrm{l} /$ well $0.001 \mathrm{Lf} / \mathrm{ml}$ diphtheria toxin diluted in complete culture medium was added and the plates were incubated at $37{ }^{\circ} \mathrm{C}$ and $5 \% \mathrm{CO}_{2}$ for $2 \mathrm{~h}$ for toxin neutralization. Subsequently, $50 \mu \mathrm{l} /$ well suspension of Vero cells were added $(12,500$ cells/well) and incubated at $37^{\circ} \mathrm{C}$ in $5 \% \mathrm{CO}_{2}$ for 6 days. The number of wells containing viable Vero cells was determined by microscopy. The neutralizing antibody titers were expressed as the $\log _{2}$ value of the highest serum dilution that was still capable of protecting the Vero cells from the challenge of diphtheria toxin.

\subsection{Statistical analysis}

Data from antibody titers and neutralizing antibody titers were analyzed by one way ANOVA with Bonferroni post-test by using GraphPad Prism software (version 5.02). A p $<0.05$ was considered to be significant.

\section{Results}

\subsection{Size and zeta potential of DT-alumNPs and adsorption efficiency of DT to alumNPs and to $\mathrm{AlPO}_{4}$}

DT-Gibbsite and DT-Boehmite were characterized in terms of particle size, polydispersity index (PDI) and zeta potential. DT-alumNPs were in the $\mu \mathrm{m}$ range, i.e. outside the measuring range of the DLS equipment, regardless the buffer composition (data not shown).

The effect of the type of particle on the zeta potential was examined. In histidine buffer, boehmite resulted in a positive surface charge, while gibbsite showed a negative zeta potential at the same concentration. When increasing the particle concentration the zeta potential became less negative for the boehmite and became positive for the gibbsite. The effect of the addition of DT on the zeta potential was also examined. It was observed that (i) addition of DT to the alumNPs formulation resulted in a lower zeta potential than alumNPs only and (ii) increasing the DT:alumNPs ratio from 1:1 to $1: 10$ by increasing the alumNP concentration resulted in a higher zeta potential, indicating a relative lower level of negatively charged DT on their surface compared with the slightly positively charged alumNPs (Table 3 ). No significant changes in zeta potential were observed within the first $24 \mathrm{~h}$ of storage (Table 3 ). The alumNPs in PB showed a more negative zeta potential than in histidine buffer.

The adsorption efficiency of DT on the alumNPs and $\mathrm{AlPO}_{4}$ at $3 \mathrm{~h}$ 
Table 3

Zeta potential of DT-alumNPs and percentages of DT adsorption on alumNPs and $\mathrm{AlPO}_{4}(\mathrm{n}=3)$. Data are average \pm SEM.

\begin{tabular}{|c|c|c|c|c|c|}
\hline \multirow[b]{3}{*}{ Formulation } & \multirow[b]{3}{*}{ Buffer } & \multicolumn{3}{|l|}{ Time (h) } & \\
\hline & & 0 & 3 & 24 & \\
\hline & & Zeta Potential (mV) & Adsorption efficiency (\%) & & \\
\hline Gibbsite $^{*}$ & $\mathrm{~PB}$ & $-24.7 \pm 0.4$ & - & - & - \\
\hline DT-Gibbsite $^{*)} 1: 1$ & & $-32.6 \pm 0.5$ & $-32.8 \pm 0.3$ & $-32.1 \pm 0.3$ & $27.1 \pm 0.5$ \\
\hline Gibbsite $^{*}$ & Histidine & $-0.9 \pm 0.3$ & - & - & - \\
\hline DT-Gibbsite $^{*}$ 1:1 & & $-27.1 \pm 0.1$ & $-27.0 \pm 0.2$ & $-26.7 \pm 0.2$ & $11.4 \pm 1.0$ \\
\hline Gibbsite $^{* * *}$ & & $17.6 \pm 0.3$ & - & - & - \\
\hline DT-Gibbsite $\left.{ }^{* * * x}\right) 1: 10$ & & $-22.8 \pm 0.3$ & $-22.5 \pm 0.2$ & $-19.0 \pm 0.6$ & $81.3 \pm 0.1$ \\
\hline Boehmite $^{*}$ & & $3.4 \pm 0.4$ & - & - & - \\
\hline DT-Boehmite $^{*}$ 1:1 & & $-23.8 \pm 0.2$ & $-23.2 \pm 0.2$ & $-25.7 \pm 0.1$ & $25.5 \pm 0.4$ \\
\hline Boehmite $\left.^{* k *}\right)$ & & $13.9 \pm 0.5$ & - & - & - \\
\hline DT-Boehmite $^{* *}$ 1:10 & & $-15.4 \pm 0.6$ & $-8.5 \pm 0.2$ & $-4.7 \pm 0.5$ & $83.2 \pm 0.1$ \\
\hline DT-AlPO $_{4}$ 1:30 & PBS & - & - & - & $38.7 \pm 0.3$ \\
\hline
\end{tabular}

*) $36 \mu \mathrm{g} / \mathrm{ml} \mathrm{Al}{ }^{3+}$.

**) $360 \mu \mathrm{g} / \mathrm{ml} \mathrm{Al}^{3+}$.

after mixing was measured (Table 3). In solutions with DT: alumNPs 1:10 ratio more than $80 \%$ DT was adsorbed to the alumNPs. For a DT: alumNPs 1:1 ratio the DT adsorption on the alumNPs was less than $40 \%$. This was also observed for the $\mathrm{AlPO}_{4}$ particles. This indicated that at equal weight ratios the alumNP surface became saturated with DT.

\subsection{Immunization study for adjuvant screening}

In an immunization study using a hMN several adjuvants were screened on their efficiency to potentiate the immune response of DT. The selected adjuvants were CpG ODN, Poly(I:C), cholera toxin, gibbsite and boehmite (the latter two in DT: alumNPs ratios of $1: 1$ and $1: 10)$.

After the prime, the groups DT-Gib 1:1 and DT-Boe 1:10 induced higher IgG titers than the DT group (Fig. 1 A). The DT-Boe 1:10 group showed even a comparable response to the positive control DT-AlPO (Fig. 1 A).

At day 42, DT-specific total IgG titers increased further for all formulations (Fig. 1 B). However, no significant adjuvant effect was observed in comparison with the DT control group (Fig. 1 B). The DT-CT group had a comparable IgG response to the positive control DT-AlPO .

At day 63 (Fig. $1 \mathrm{C}$ ), the response of all the groups was very close to the response of positive control DT-AlPO ${ }_{4}$, despite a 15 fold lower dose (Fig. 1 C). However, the addition of different adjuvants did not enhance further the response evoked by DT after three vaccinations (Fig. $1 \mathrm{C}$ ).

Besides the IgG total response, the IgG1 and IgG2a responses were also measured. IgG1 and IgG2a ratios were not dependent on adjuvant type (Supplementary material, Fig. S1) ( $>$ > 0.05). This indicates that the Th2/Th1 balance was not influenced by the adjuvants.

The functionality of the antibody response was investigated by determining toxin neutralizing antibody titers in serum on day 63. The positive control DT-ALPO 4 showed higher levels of toxin-neutralizing antibodies than all other groups ( $\mathrm{p}<0.05$ ) (Fig. 2). Overall, the addition of an adjuvant to DT did not improve the functional response. Similarly to the IgG total titers, DT-Gibbsite 1:10 was less immunogenic as compared to plain DT.

Based on neutralizing antibody results and the primary response, poly(I:C) and gibbsite (the formulation in 1:1 ratio with DT) were selected as adjuvants for the studies using dMNs.

\subsection{Dissolving microneedle: Characterization and interaction with the skin}

dMN arrays were prepared with DT in absence or presence of the selected adjuvant. Very sharp dMNs containing either DT, DT-PI or DTGib could be prepared (Fig. 3) in a reproducible manner. The DT content (full dose or fractional dose) did not affect the shape either (Fig. 3 and data not shown).

Hyaluronan, DT and gibbsite were uniformly distributed within the $\mathrm{dMN}$ as investigated with confocal 3D imaging using fluorescently labelled components (Fig. 4).

dMN arrays prepared from full dose of either DT, DT-PI or DT-Gib were applied on ex vivo human skin. After withdrawal and application of trypan blue, the number of blue spots were determined and the penetration efficiency and the standard deviation were calculated being respectively $95.8 \pm 7.2 \%, 89.6 \pm 9.5 \%$ and $100.0 \pm 0.0 \%$, respectively $(\mathrm{n}=3)$.

As shown in Fig. 3, dMNs with DT or DT-PI incorporated dissolved completely in the skin within $20 \mathrm{~min}(100 \pm 0 \%$ dissolved MN volume, mean $\pm \mathrm{sd}$ ), the dMNs with DT-Gib incorporated resulted in some dMN leftover (approximately $91 \pm 1 \%$ dissolved volume, mean \pm sd).

After 20 min microneedle dissolution in fresh human skin and withdrawal of the remaining $\mathrm{dMN}$ array, the hyaluronan, DT and gibbsite were visualized in the skin by CLSM during $5 \mathrm{~h}$ each $30 \mathrm{~min}$ as function of depth parallel to the skin surface (Fig. 5, only 0,3 and $5 \mathrm{~h}$ time points are shown). All components were deposited and co-localized in the epidermis and top layers of the dermis. Furthermore, the delivered antigen without or with gibbsite remained at the site of administration for at least $5 \mathrm{~h}$, whereas HA is mostly diffused away after $3 \mathrm{~h}$ or less.

Finally, the amount of DT delivered into the skin after dMN array application using infrared labelled DT was determined. After $20 \mathrm{~min}$ of application in the skin, the dMNs containing a full or $1 / 3$ dose of DT delivered respectively $0.32 \pm 0.02 \mu \mathrm{g}$ and $0.13 \pm 0.04 \mu \mathrm{g}$ of DT in ex vivo human skin and $0.35 \pm 0.03 \mu \mathrm{g}$ and $0.10 \pm 0.01 \mu \mathrm{g}$ of DT in $e x$ vivo mouse skin (mean $\pm \mathrm{sd}, \mathrm{n}=3$ ).

\section{4. dMN arrays and hMNs: single-full dose vs. repeated-fractional dose}

The aim of this study was to compare RFrD with SFD administration of DT with or without adjuvant using dMN arrays and a hMN keeping the total dose of DT and antigen approximately the same.

Following prime vaccination by a hMN, IgG titers after DT RFrD were higher than DT SFD (Fig. 6 A). To determine whether MN piercing itself has an effect on the immune response, a hMN group (PBS/PBS/DT SFD) consisting of two consecutive days of PBS injection and a third day of SFD DT injection was included. As the IgG titers were higher than that of the SFD injection, microneedle piercing as such seems to enhance the immune response.

Prime administration of DT by dMNs gave higher IgG responses than hMN injection (Fig. 6 A). DT RFrD by dMNs did not significantly increase the IgG levels compared to DT SFD by dMNs, although there is a 
A

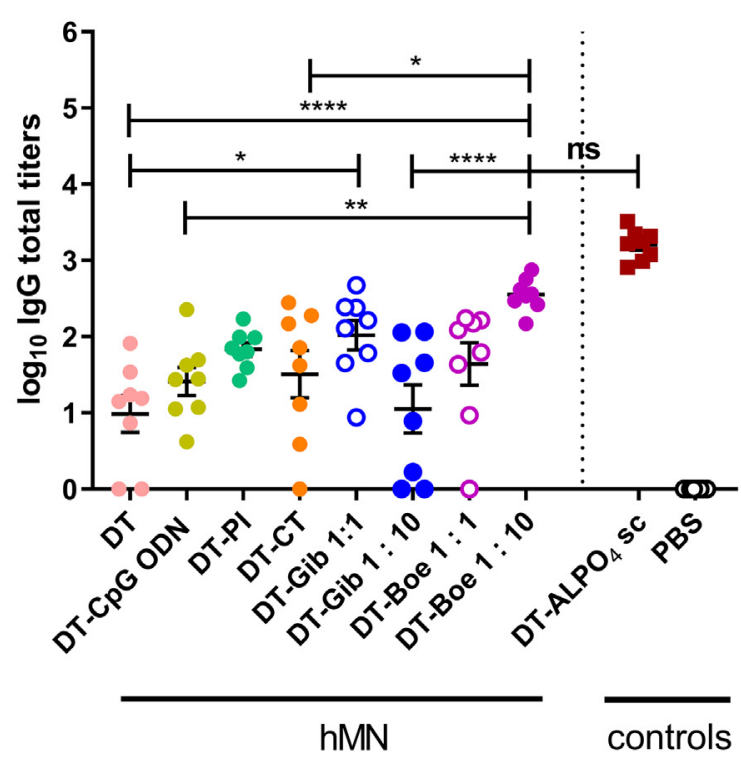

B

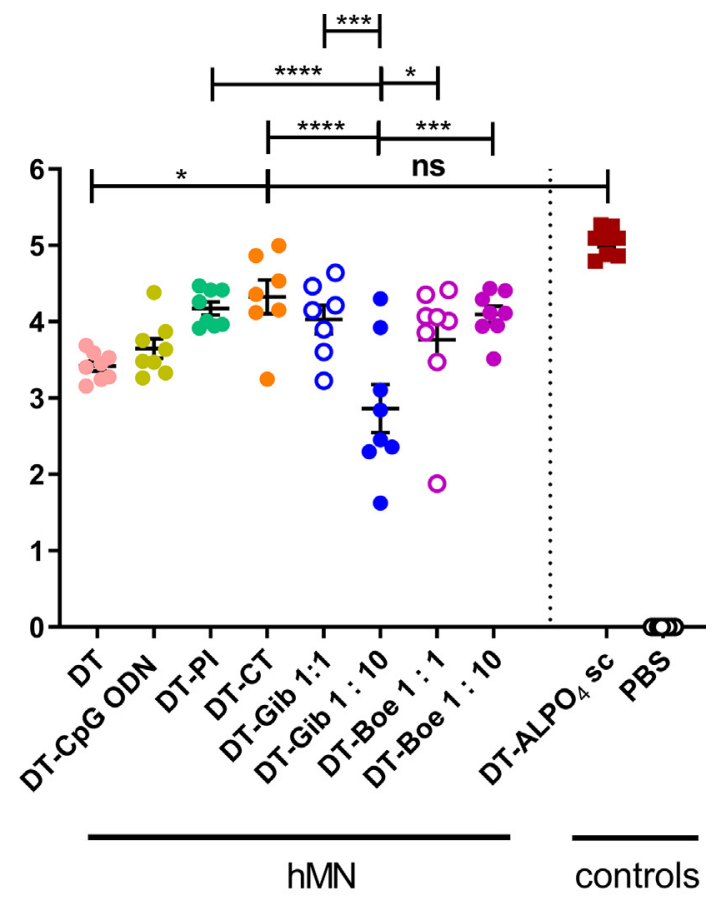

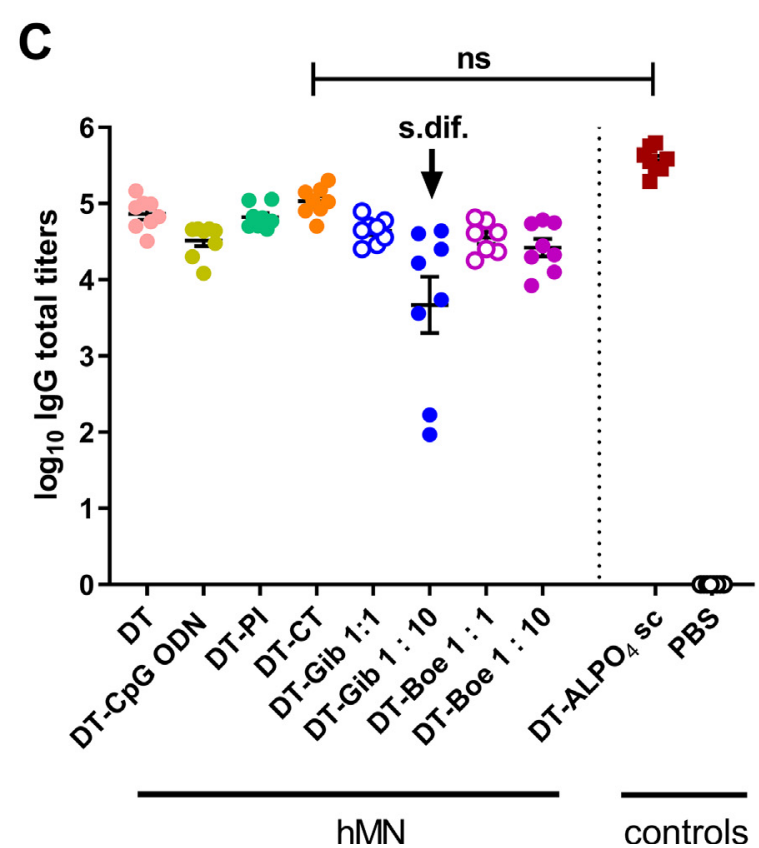

Fig. 1. DT-specific total IgG titers on day 21 (A), 42 (B) and 63 (C). After each vaccination, the $\mathrm{AlPO}_{4}$ was significantly different from each other group except the no significant (ns) group reported. After 63 days, the titers in the DT-Gib 1:10 group was significantly lower (s.dif.) compared to the titers in all the other groups. Bars represent mean \pm SEM, $\mathrm{n}=8$. ${ }^{*} \mathrm{p}<0.05,{ }^{* * \mathrm{p}}<0.01,{ }^{*}{ }^{*} \mathrm{p} p<0.001$ and ${ }^{* * * * \mathrm{p}}<0.0001$. DT dose was $0.36 \mu \mathrm{g}$ except DT-AlPO ${ }_{4}$ sc: $5 \mu \mathrm{gg}$. hMN: hollow microneedle; DT: diphtheria toxoid; PI: poly(I:C); CT: cholera toxin; Gib: gibbsite; Boe: boehmite; $\mathrm{AlPO}_{4}$ : aluminum phosphate.

trend of a higher response and after RFrD there is less variation in the response. Additionally, the application of empty dMN arrays in the first two days and the SFD administration of the DT on the third day (E/E/ DT SFD) showed a comparable IgG response as RFrD. The encapsulation of an adjuvant (poly(I:C) or gibbsite) together with DT in the dMNs, did not increase the DT-specific total IgG response when delivered as SFD or RFrD compared to the absence of an adjuvant. Finally, the administration of DT by dMNs gave a higher response than the control of DTpoly(I:C) injected subcutaneously and a response comparable to the positive control, DT-ALPO 4 sc, with a 15 times higher DT dose. Conversely, the hMN groups showed comparable response to the control of
DT-PI sc and a significantly lower response than the positive control DT-ALPO 4 sc.

After the first boost, DT-specific total IgG titers increased (Fig. 6 B) but the differences between the groups were similar as after the prime with a few changes. DT RFrD by dMNs was comparable to DT RFrD using hMN but still higher than PBS/PBS/DT SFD hMN group. Furthermore, the positive controls DT-PI and DT-AlPO ${ }_{4}$ subcutaneously injected were comparable to all hMN and dMNs groups except to DT SFD hMN and DT SFD hMN and PBS/PBS/DT SFD hMN groups, respectively, which showed a significant lower response.

After the second boost (Fig. $6 \mathrm{C}$ ) the titers of most groups further 


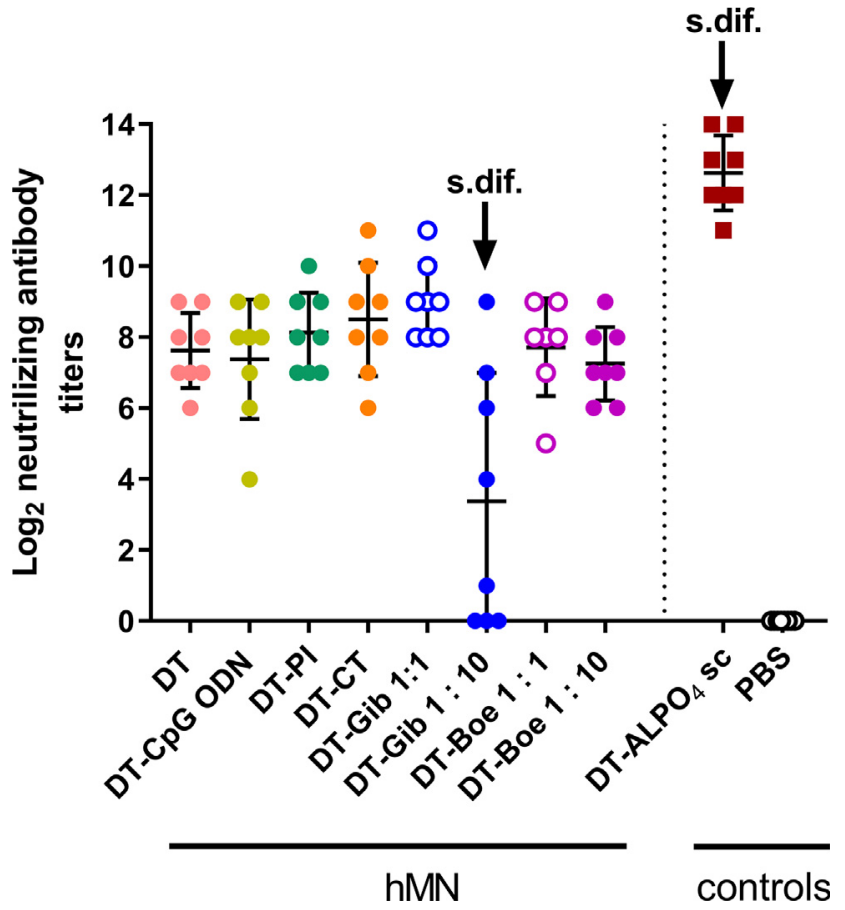

Fig. 2. DT-neutralizing antibody titers. Results are shown for serum collected on day 63. The titers in the DT-Gib 1:10 group and the DT-AlPO4 group were, respectively, significantly lower or higher (s.dif.) from the titers in all the other groups. Bars represent mean \pm SEM, $\mathrm{n}=8$. hMN: hollow microneedle; DT: diphtheria toxoid; PI: poly(I:C); CT: cholera toxin; Gib: gibbsite; Boe: boehmite; $\mathrm{AlPO}_{4}$ : aluminum phosphate.

increased, although slightly. Apparently a plateau in the immune response was reached. After this second boost, for the first time the DT RFrD dMNs group developed a higher response than E/E/DT SFD dMNs.

The IgG1/IgG2a ratios are depicted in Fig. S2 in Supplementary material. DT vaccination by dMNs and addition of gibbsite or poly(I:C) as adjuvant modestly changed the IgG1/IgG2a ratio, shifting the balance slightly to Th1.

High levels of toxin-neutralizing antibodies were induced after vaccination by means of dMNs, regardless the dosing modality or the presence of an adjuvant, and by DT RFrD by hMN (Fig. 7). No adjuvant effect was observed after addition of PI in dMNs (DT SFD dMNs gave a similar response compared to DT-PI SFD dMNs and DT-PI SFD sc), but DT-PI RFrD dMNs resulted higher in response compared to DT-PI SFD dMNs and DT-PI SFD sc. In this case, the dosing modality made a difference in protection against the toxin, resulting a RFrD of DT-PI in a much higher toxin neutralization.

\section{Discussion}

\subsection{Repeated-fractional doses effect}

The aim of this study was to obtain insight in whether dermal vaccination by RFrD of DT enhances the specific IgG response compared to that after SFD antigen dermal administration. The present study corroborated existing data (Schipper et al., 2016a; Johansen et al., 2008) by showing a superior response by RFrD compared to SFD of antigen after vaccination by hMN. In the present study less consecutive days of administration were used as in the previous studies: $33 \%$ in each consecutive day during 3 days vs 4,7 and 8 days in the previous studies. Furthermore, low responders seemed to benefit more from the $\mathrm{RFrD}$ regime and it was demonstrated that consecutive skin piercing by hMN (PBS/PBS/DT SFD) could enhance the immune response compared to a DT SFD only by hMN. Piercing of the skin may cause some

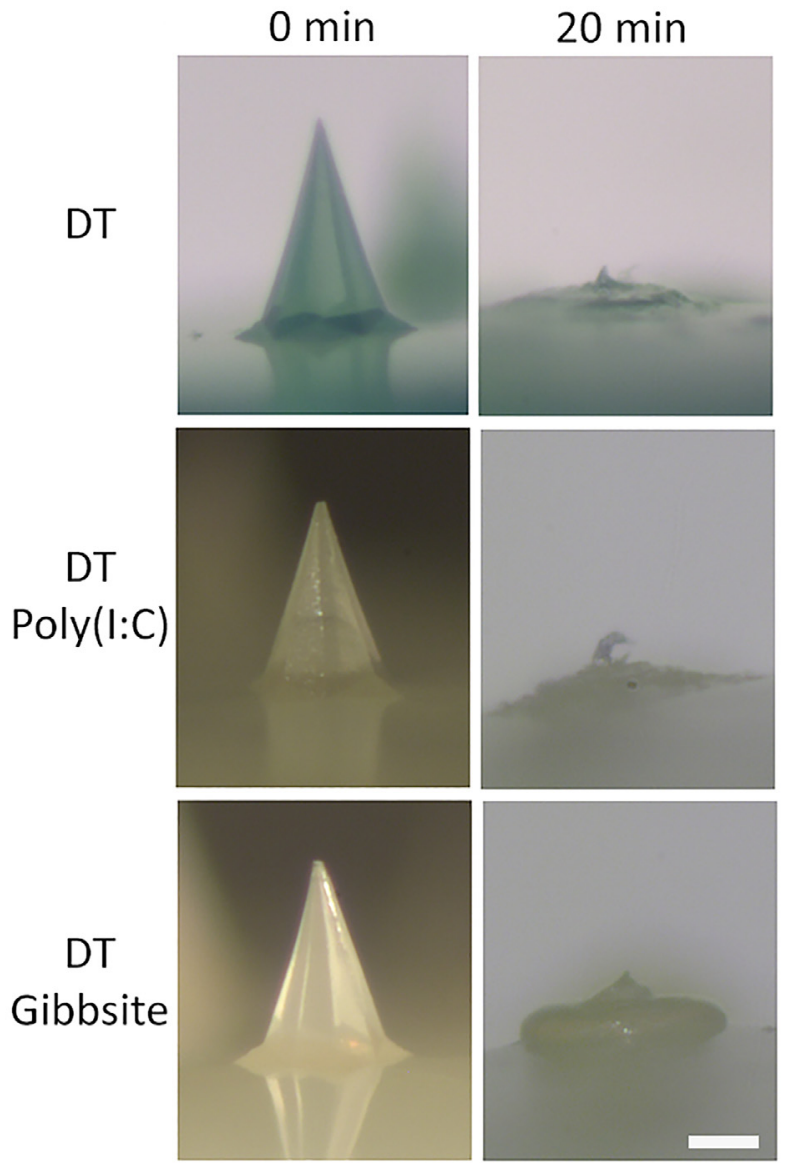

Fig. 3. Brightfield microscope images $(5 x)$ of microneedles with DT, DT-PI and DT-Gib full dose content before application on the skin ( $0 \mathrm{~min}$ ) and after $20 \mathrm{~min}$ dissolution into ex vivo human skin. Scale bar $100 \mu \mathrm{m}$.
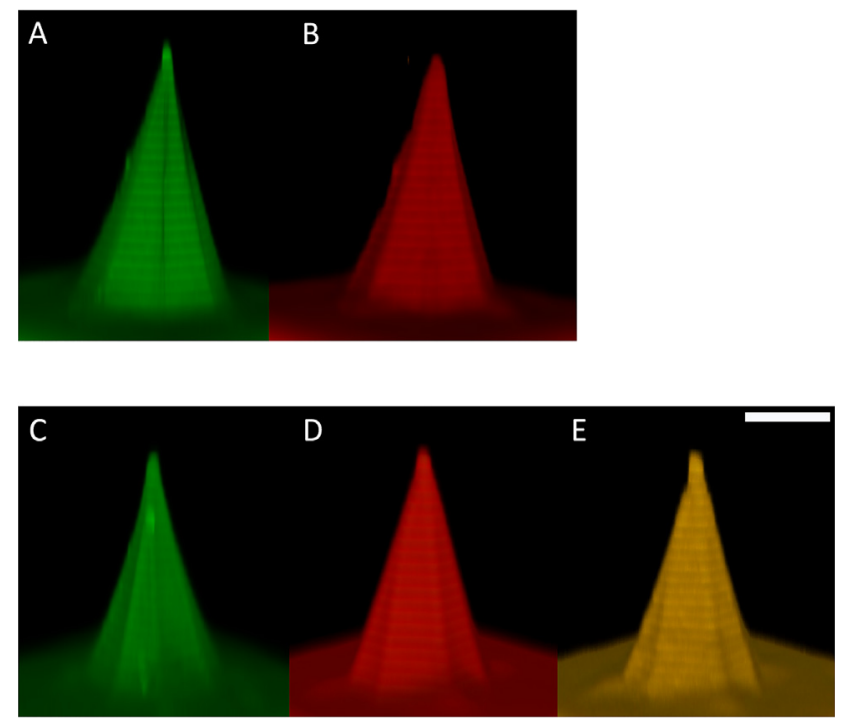

Fig. 4. CLSM 3D images of the distribution of fluorescently labelled HA (A) and DT (B) in the hyaluronan-based dMNs containing DT; Fluorescently labelled HA (C), DT (D) and Gib (E) in hyaluronan-based dMNs containing DT adsorbed gibbsite nanoparticles. Both dMNs (A-B and C-E) contained a full dose of DT or DT-Gib. Scale bar $100 \mu \mathrm{m}$.

cell death or other local damage resulting in the release of damageassociated molecular patterns (DAMPs) and subsequent attraction of antigen presenting cells to the immunization area (Depelsenaire et al., 
$\mathbf{O h}$
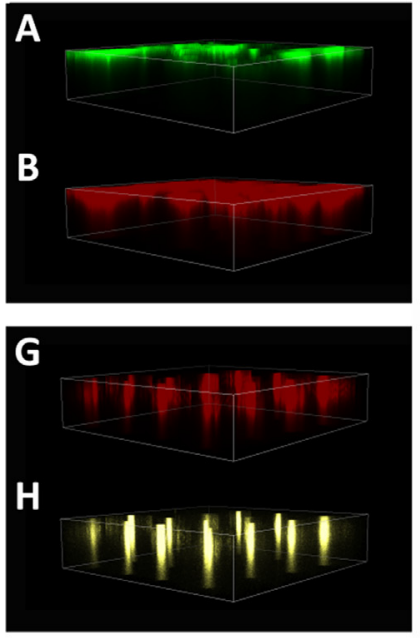

$3 \mathrm{~h}$
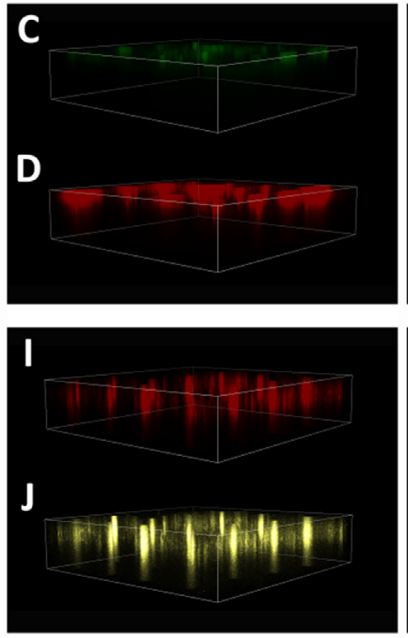

$5 \mathrm{~h}$
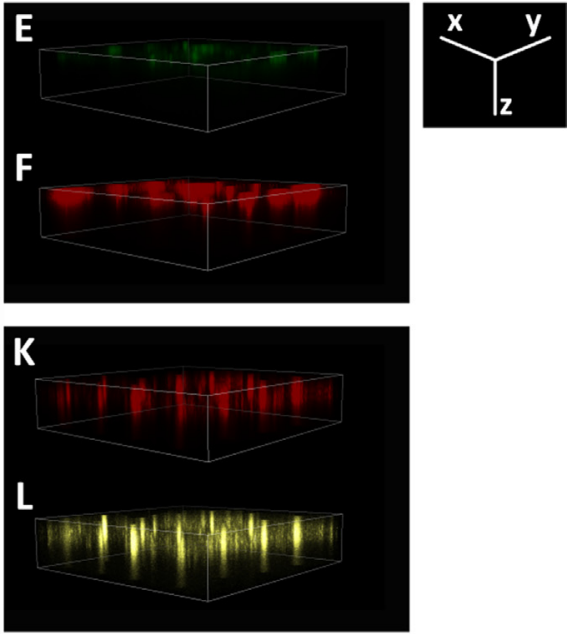

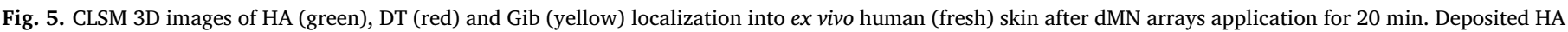

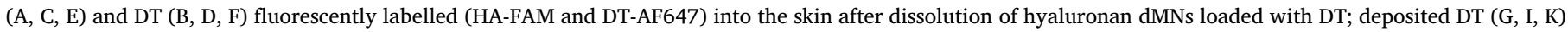

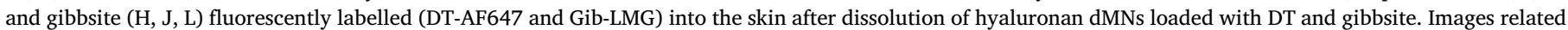

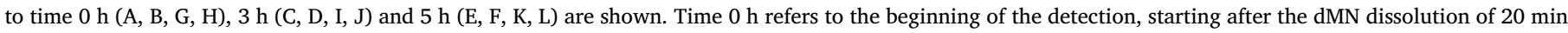
and removal of the dMN array. The location of the skin surface is the xy area on top of the z-axis representing the depth of imaging ( 0.7 mm).

2014). However, application of empty dMN arrays (E/E/DT SFD), inducing an even higher number of piercings in the skin compared to a hMN (16 dMNs per array vs $1 \mathrm{hMN}$ ) and applied by a 20 min pressure on the skin potentially leading to skin inflammation (Depelsenaire et al., 2014; Meliga et al., 2013). This did not enhance the response further compared to a SFD antigen administration by dMNs. This may be explained by a maximal level of immunity reached after prime for the E/E/DT SFD group due to the skin piercing by the first empty array application inducing release of DAMPs and then an immune response with no additional further effect after application of the other empty and then DT loaded dMN arrays.

\section{2. dMNs vs a $h M N$}

Vaccination by means of dMNs led to significantly higher response than by hMN, as already reported in our previous study (Leone et al., 2019), showing a faster increase in DT-specific IgG responses already after the first immunization. This can be related to several factors. First, the use of different MN types: a hMN injects the vaccine at a specific intradermal depth point $(120 \mu \mathrm{m})$ while the longer dMNs $(300 \mu \mathrm{m})$ release the antigen at various skin depths simultaneously possibly reaching a higher number of immune cells and involving different immune cell populations (Matsuo et al., 2014), although in literature no difference in the immune response is reported when comparing different injection depths (Schipper et al., 2016b). Second, the number of needles piercing the skin ( $16 \mathrm{dMNs}$ vs $1 \mathrm{hMN}$ ) and the pressure applied on the skin (20 min for the dMN array application and no pressure for the hMN) potentially causing inflammation and thus more release of DAMPs in a larger region, facilitating attraction of antigen presenting cells. Third, the presence of low molecular weight species of HA. Although high MW HA is considered immunologically inert, low molecular weight HA fragments, potentially present in the dMNs or generated in vivo, can elicit various proinflammatory responses leading to innate immune activation (Termeer et al., 2002; Termeer et al., 2000), although this is not observed in recent studies in our group (Leone et al., 2020) and in literature (Oh et al., 2010). Fourth, the prolonged exposure of the antigen during dMN dissolution to relevant immune cells may enhance the response (Gatto et al., 2007). Fifth, the presence of low responding and no-responding mice after prime vaccination in SFD by hMN: in a previous study (Schipper et al., 2017) and in the adjuvants screening of the present study, the titers after DT vaccination by hMN resulted in an overall higher response being closer to the positive control DT-AlPO 4 than in the SFD vs RFrD immunization study.

\subsection{Adjuvants vs repeated-fractional dosing by $d M N s$}

Antigen dose sparing can be achieved with the use of adjuvants (Ding et al., 2009; Reed et al., 2013). Besides adjuvants (CpG, PI and CT) commonly used for experimental dermal vaccination (Ding et al., 2009; Du et al., 2018; Leone et al., 2017), aluminum-based NPs (alumNPs), so far tested only for subcutaneous or intramuscular administration (Crepeaux et al., 2015; Reed et al., 2013; Gupta, 1998), were included for intradermal vaccination.

The selection, in the first immunization study by hMN, of the optimal adjuvants for DT vaccination by dMNs led to the choice of poly $(\mathrm{I}: \mathrm{C})$ and gibbsite. Poly(I:C) gave a more robust response after primary immunization, similar to CT, than other adjuvants and it has a better feasibility for human vaccination than CT. The selection of gibbsite (in ratio 1:1 with DT) was based on the following considerations: (i) its higher response among the DT adjuvanted with alumNPs and (ii) dermal injection of gibbsite, as of boehmite too, did not induce any palpable persistent intradermal injection-site nodules in mice, as previously observed after intradermal injection of classical aluminum preparations (Vogelbruch et al., 2000).

The addition of adjuvants for intradermal vaccination by both hMN and dMNs did not enhance the IgG total levels further compared to unadjuvanted antigen indicating that the response reached already a plateau and any extra did not lead to a higher response. However, vaccination by dMNs, in the presence of poly(I:C) or gibbsite, shifted the Th2/Th1 balance to Th1. This was not observed using hMNs. This change in response may be related to the depot created in the skin after $\mathrm{dMN}$ dissolution leading to a sustained release of antigen and adjuvant (Gatto et al., 2007).

In our study, similarly potent DT-specific IgG responses were observed upon intradermal vaccination by dMNs of SFD, RFrD and adjuvanted DT. This demonstrates that by using dMNs the immune response can be enhanced and the use of an adjuvant or prolonged antigen delivery by fractional dosing can be avoided, at least for potent antigens. An adjuvant-free vaccine would then avoid side-effects and potential stability problems related to the presence of the adjuvant 
A

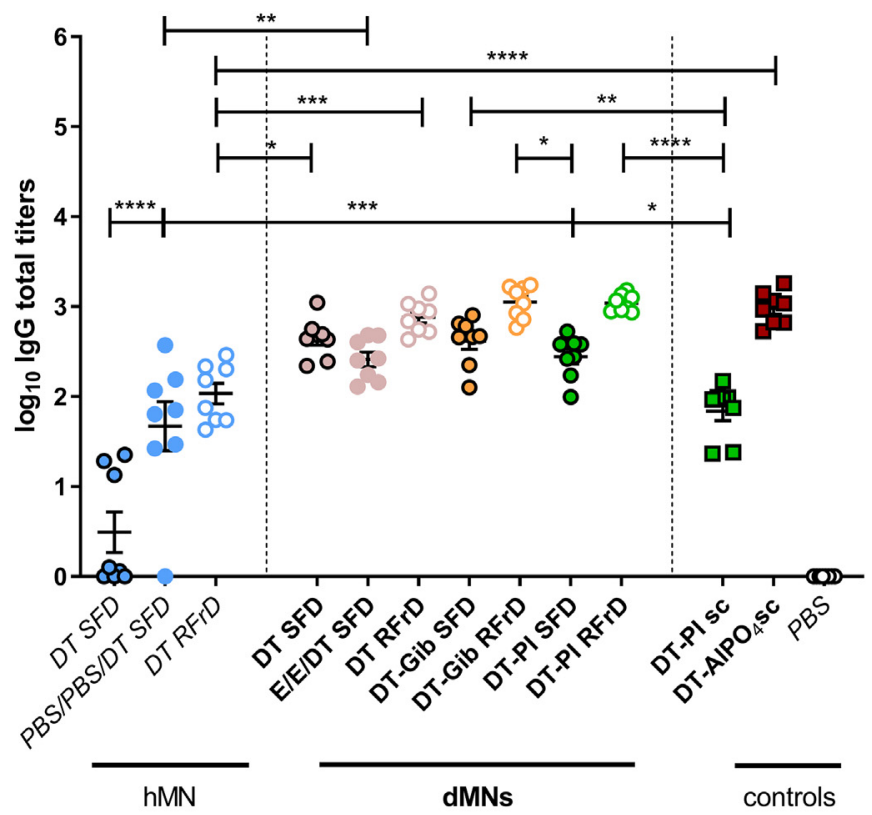

hMN

dMNs

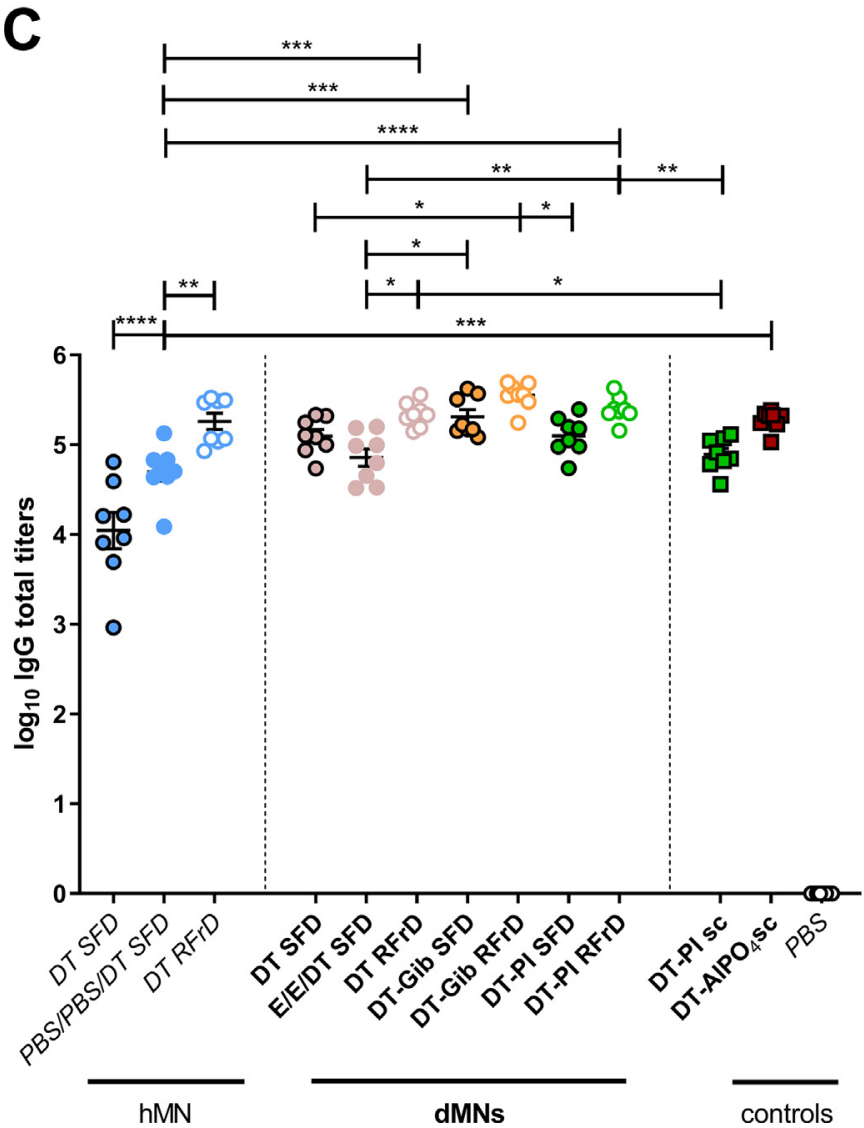

Fig. 6. DT-specific total IgG antibody titers measured in BALB/c mice on day 21 (A), day 42 (B) and day 63 (C). Bars represent mean \pm SEM, $n=8$. *p $<0.05$, $* * \mathrm{p}<0.01,{ }^{* * * \mathrm{p}}<0.001, * * * * \mathrm{p}<0.0001$. SFD: single-full dose; RFrD: repeated-fractional dose; dMNs: dissolving microneedles; hMN: hollow microneedle; DT: diphtheria toxoid; PI: poly(I:C); Gib: gibbsite; E: empty dMNs; $\mathrm{AlPO}_{4}$ : aluminum phosphate. 


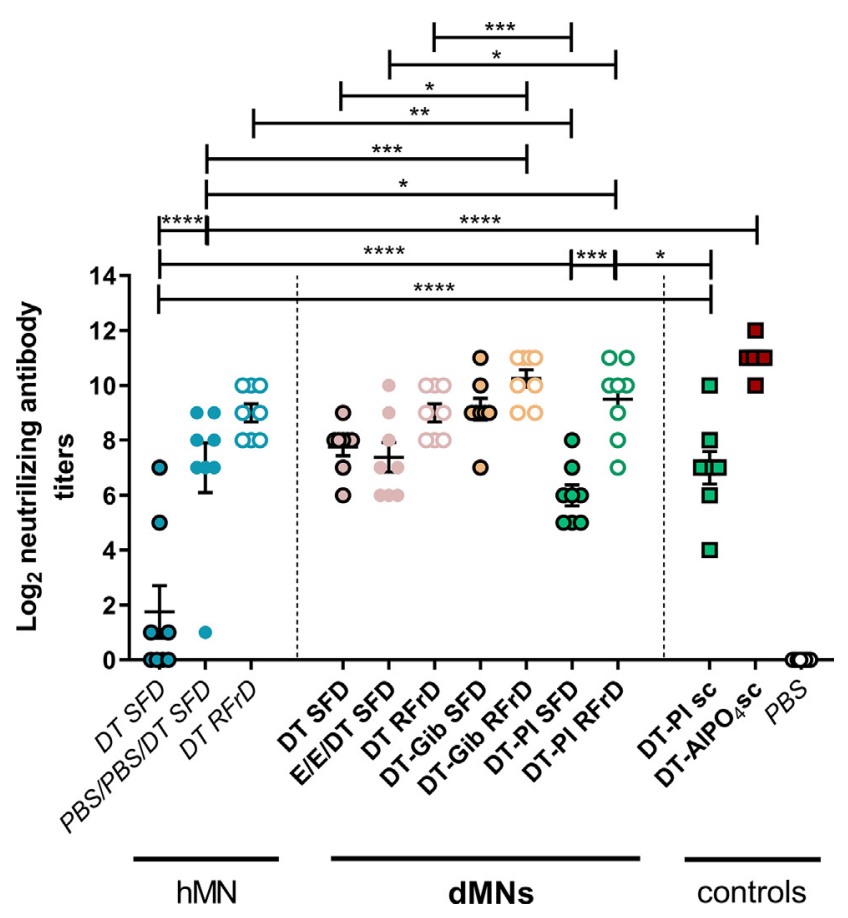

Fig. 7. DT-neutralizing antibody titers. Results are shown for serum collected on day 63. Bars represent mean \pm SEM, $\mathrm{n}=8$. * $\mathrm{p}<0.05$, **p $<0.01$, $* * * \mathrm{p}<0.001, * * * * \mathrm{p}<0.0001$. SFD: single-full dose; RFrD: repeated-fractional dose; dMNs: dissolving microneedles; hMN: hollow microneedle; DT: diphtheria toxoid; PI: poly(I:C); Gib: gibbsite; E: empty dMNs; $\mathrm{AlPO}_{4}$ : aluminum phosphate.

(Kumru et al., 2014; Mbow et al., 2010).

However, results from neutralizing antibody assay indicated a higher protection against diphtheria toxin for adjuvanted-DT RFrD dMNs compared to unadjuvanted DT in dMNs, hMN groups and even conventional injection of DT-PI. This may be related to a prolonged exposure of the antigen during and after dMN dissolution to relevant immune cells involved in the humoral protection as mentioned above.

Overall these observations lead to the conclusion that the combination of RFrD and adjuvant in dMNs, but not their separated use, can be very efficient in respect to the antibody response and the neutralization of the diphtheria toxin effect.

Finally, Joyce et al. (2019) reported how extended-delivery vaccination by means of dMNs enables a single vaccination to generate immune responses equivalent to a two-dose vaccination regimen for vaccines as IPV, tetanus toxoid and influenza but the same extendeddelivery vaccination does not enhance immune response to the liveattenuated measles vaccine. This demonstrates that RFrD of the antigen can lead to a superior immune response than SFD depending on the antigen type.

\section{4. dMNs vs conventional subcutaneous injections}

This study focused on minimally-invasive delivery for DT immunization by dMNs and it has been shown that vaccination by dMNs loading unadjuvanted DT led to comparable responses already after prime immunization as compared to invasive subcutaneous injections of DT-AlPO4 with almost 15 and 415 times higher DT and adjuvant doses respectively. These results corroborated existing data in literature where a comparable or even higher response was obtained after vaccination by dMNs than conventional subcutaneous injection (Bachy et al., 2013; Edens et al., 2015; Matsuo et al., 2014; Pattani et al., 2011; Zhu et al., 2016) and similar response was shown after vaccination with hMN in comparison with the above mentioned positive control (Du et al., 2018; Schipper et al., 2017). Higher IgG responses after prime and comparable responses after the two boost immunizations demonstrate the efficiency of a dermal vaccination by dMNs compared to subcutaneous injection of adjuvanted-DT (DT-PI sc).

\subsection{Characterization of the alumNPs}

Fabrication of dMN arrays and dMN interaction with the skin, regardless the formulation, was successful as previously demonstrated (Leone et al., 2019). However, dissolution of DT-Gib was not complete after $20 \mathrm{~min}$. This may be related to the particle aggregation observed in the alumNPs characterization studies. Aggregation is likely due to the $\mathrm{pH}$, as the alumNPs are stable at $\mathrm{pH}<6$ and aggregate above this value (H. Vrieling et al, manuscript in preparation).

Boehmite and gibbsite were characterized in the transmission electron microscope for their shape and size resulting in $\mathrm{nm}$ range (Buining et al., 1994; Verhoeff et al., 2011). In the present study their size resulted in the $\mu \mathrm{m}$ range using dynamic light scattering (data not shown). This suggests that particle aggregation occurred. Besides the presence of DT and the concentration of alumNPs, the buffer composition also had a role on the zeta potential: PB lowered the zeta potential more than the histidine buffer. A possible explanation can be found in the phosphate groups from PB exchanging with hydroxyl groups of alumNPs so that a mixture of aluminum oxyhydroxide and aluminum phosphate is obtained.

\section{Conclusion}

In conclusion, when using hMN a delivery of the antigen over multiple days can enhance the immune response more than a SFD delivery. The SFD vaccination by dMNs can enhance a much higher response than hMN, however fractional delivery administration by using dMNs does not lead to a superior response. Moreover, dMNs demonstrate no further increase in response by co-encapsulation of the adjuvant and a comparable or even higher response than, respectively, the current benchmarks: AlPO4 adsorbed DT and DT-PI administered subcutaneously. These findings demonstrate the potential of dMNs as vaccine delivery device addressing to a SFD administration of an adjuvant-free vaccine to have a fast and high functional response.

\section{CRediT authorship contribution statement}

M. Leone: Conceptualization, Investigation, Formal analysis, Data curation, Writing - review \& editing. S. Romeijn: Investigation. G. Du: Investigation. S.E. Le Dévédec: Investigation. H. Vrieling: Conceptualization. C. O’Mahony: Resources. J.A. Bouwstra: Conceptualization, Supervision. G. Kersten: Conceptualization, Supervision.

\section{Declaration of Competing Interest}

The authors declare that they have no known competing financial interests or personal relationships that could have appeared to influence the work reported in this paper.

\section{Acknowledgements}

Part of this work was funded by Intravacc.

We thank Amy Kogelman from Intravacc for her help with the neutralizing antibody assay.

\section{Appendix A. Supplementary material}

Supplementary data to this article can be found online at https:// doi.org/10.1016/j.ijpharm.2020.119182. 


\section{References}

Bachy, V., Hervouet, C., Becker, P.D., Chorro, L., Carlin, L.M., Herath, S., Papagatsias, T., Barbaroux, J.B., Oh, S.J., Benlahrech, A., Athanasopoulos, T., Dickson, G., Patterson, S., Kwon, S.Y., Geissmann, F., Klavinskis, L.S., 2013. Langerin negative dendritic cells promote potent CD8 + T-cell priming by skin delivery of live adenovirus vaccine microneedle arrays. Proc. Natl. Acad. Sci. USA 110, 3041-3046.

Buining, P.A., Pathmamanoharan, C., Jansen, J.B.H., Lekkerkerker, H.N.W., 1991. Preparation of colloidal boehmite needles by hydrothermal treatment of aluminum alkoxide precursors. J. Am. Ceram. Soc. 74, 1303-1307.

Buining, P.A., Philipse, A.P., Lekkerkerker, H.N.W., 1994. Phase-behavior of aqueous dispersions of colloidal boehmite rods. Langmuir 10, 2106-2114.

Crepeaux, G., Eidi, H., David, M.O., Tzavara, E., Giros, B., Exley, C., Curmi, P.A., Shaw, C.A., Gherardi, R.K., Cadusseau, J., 2015. Highly delayed systemic translocation of aluminum-based adjuvant in CD1 mice following intramuscular injections. J. Inorg. Biochem. 152, 199-205.

de Belder, A.N., Wik, K.O., 1975. Preparation and properties of fluorescein-labelled hyaluronate. Carbohydr. Res. 44, 251-257.

Depelsenaire, A.C.I., Meliga, S.C., McNeilly, C.L., Pearson, F.E., Coffey, J.W., Haigh, O.L., Flaim, C.J., Frazer, I.H., Kendall, M.A.F., 2014. Colocalization of cell death with antigen deposition in skin enhances vaccine immunogenicity. J, Invest. Dermatol, 134, 2361-2370.

Ding, Z., Van Riet, E., Romeijn, S., Kersten, G.F., Jiskoot, W., Bouwstra, J.A., 2009. Immune modulation by adjuvants combined with diphtheria toxoid administered topically in BALB/c mice after microneedle array pretreatment. Pharm. Res. 26, 1635-1643.

Du, G., Leone, M., Romeijn, S., Kersten, G., Jiskoot, W., Bouwstra, J.A., 2018. Immunogenicity of diphtheria toxoid and poly(I:C) loaded cationic liposomes after hollow microneedle-mediated intradermal injection in mice. Int. J. Pharm. 547, 250-257.

Edens, C., Collins, M.L., Goodson, J.L., Rota, P.A., Prausnitz, M.R., 2015. A microneedle patch containing measles vaccine is immunogenic in non-human primates. Vaccine $33,4712-4718$.

Gatto, D., Martin, S.W., Bessa, J., Pellicioli, E., Saudan, P., Hinton, H.J., Bachmann, M.F., 2007. Regulation of memory antibody levels: the role of persisting antigen versus plasma cell life span. J. Immunol. 178, 67-76.

Gill, H.S., Prausnitz, M.R., 2007. Coated microneedles for transdermal delivery. J. Control. Release 117, 227-237.

Gupta, R.K., 1998. Aluminum compounds as vaccine adjuvants. Adv. Drug Deliv. Rev. 32, 155-172.

Jiang, H., Wang, Q., Sun, X., 2017. Lymph node targeting strategies to improve vaccination efficacy. J. Control. Release 267, 47-56.

Johansen, P., Storni, T., Rettig, L., Qiu, Z., Der-Sarkissian, A., Smith, K.A., Manolova, V., Lang, K.S., Senti, G., Mullhaupt, B., Gerlach, T., Speck, R.F., Bot, A., Kundig, T.M., 2008. Antigen kinetics determines immune reactivity. Proc. Natl. Acad. Sci. USA 105, 5189-5194.

Joyce, J.C., Sella, H.E., Jost, H., Mistilis, M.J., Esser, E.S., Pradhan, P., Toy, R., Collins, M.L., Rota, P.A., Roy, K., Skountzou, I., Compans, R.W., Oberste, M.S., Weldon, W.C., Norman, J.J., Prausnitz, M.R., 2019. Extended delivery of vaccines to the skin improves immune responses. J. Control. Release 304, 135-145.

Kim, Y.C., Park, J.H., Prausnitz, M.R., 2012. Microneedles for drug and vaccine delivery. Adv. Drug Deliv. Rev. 64, 1547-1568.

Kumru, O.S., Joshi, S.B., Smith, D.E., Middaugh, C.R., Prusik, T., Volkin, D.B., 2014. Vaccine instability in the cold chain: mechanisms, analysis and formulation strategies. Biologicals 42, 237-259.

Larraneta, E., McCrudden, M.T., Courtenay, A.J., Donnelly, R.F., 2016. Microneedles: a new frontier in nanomedicine delivery. Pharm. Res. 33, 1055-1073.

Leone, M., Monkare, J., Bouwstra, J.A., Kersten, G., 2017. Dissolving microneedle patches for dermal vaccination. Pharm. Res.

Leone, M., Priester, M.I., Romeijn, S., Nejadnik, M.R., Monkare, J., O’Mahony, C., Jiskoot, W., Kersten, G., Bouwstra, J.A, 2019. Hyaluronan-based dissolving microneedles with high antigen content for intradermal vaccination: Formulation, physicochemical characterization and immunogenicity assessment. Eur J Pharm Biopharm 134, 49-59.

Leone, Mara, Romeijn, Stefan, Slütter, Bram, O'Mahony, Conor, Kersten, Gideon, Bouwstra, Joke A., et al., 2020. Hyaluronan molecular weight: Effects on dissolution time of dissolving microneedles in the skin and on immunogenicity of antigen. Eur J Pharm Sci 146. https://doi.org/10.1016/j.ejps.2020.105269.

Leone, M., van Oorschot, B.H., Nejadnik, M.R., Bocchino, A., Rosato, M., Kersten, G. O'Mahony, C., Bouwstra, J., van der Maaden, K., 2018b. Universal applicator for digitally-controlled pressing force and impact velocity insertion of microneedles into skin. Pharmaceutics 10.

Li, N., Peng, L.H., Chen, X., Nakagawa, S., Gao, J.Q., 2011. Transcutaneous vaccines: Novel advances in technology and delivery for overcoming the barriers. Vaccine 29, 6179-6190.

Matsuo, K., Yokota, Y., Zhai, Y., Quan, Y.S., Kamiyama, F., Mukai, Y., Okada, N., Nakagawa, S., 2014. A low-invasive and effective transcutaneous immunization system using a novel dissolving microneedle array for soluble and particulate antigens (vol 161, pg 10, 2012). J. Control. Release 184 9-9.

Mbow, M.L., De Gregorio, E., Valiante, N.M., Rappuoli, R., 2010. New adjuvants for human vaccines. Curr. Opin. Immunol. 22, 411-416.

Meliga, S.C., Flaim, C., Veidt, M., Kend, M.A.F., 2013. The mechanical stress caused by micro-projection arrays penetrating the skin for vaccine delivery. Aust. J. MultiDiscipl. Eng. 10.

Mile, I., Svensson, A., Darabi, A., Mold, M., Siesjo, P., Eriksson, H., 2015. Al adjuvants can be tracked in viable cells by lumogallion staining. J. Immunol. Methods 422, 87-94.

Oh, E.J., Park, K., Kim, K.S., Kim, J., Yang, J.A., Kong, J.H., Lee, M.Y., Hoffman, A.S., Hahn, S.K., 2010. Target specific and long-acting delivery of protein, peptide, and nucleotide therapeutics using hyaluronic acid derivatives. J. Control. Release 141 $2-12$.

Pattani, A., McKay, P.F., Donnelly, R.F., Garland, M.J., Migalska, K., Cassidy, C.M., Malcolm, R., Shattock, R.J., Curran, R.M., 2011. Microneedle mediated intraderma delivery of adjuvanted recombinant HIV-1 CN54gp140 effectively primes mucosal boost inoculations. AIDS Res. Hum. Retroviruses 27 A69-A69.

Peek, L.J., Middaugh, C.R., Berkland, C., 2008. Nanotechnology in vaccine delivery. Adv. Drug Deliv. Rev. 60, 915-928.

Reed, S.G., Orr, M.T., Fox, C.B., 2013. Key roles of adjuvants in modern vaccines. Nat. Med. 19, 1597-1608.

Schipper, P., van der Maaden, K., Romeijn, S., Oomens, C., Kersten, G., Jiskoot, W., Bouwstra, J., 2016. Repeated fractional intradermal dosing of an inactivated polio vaccine by a single hollow microneedle leads to superior immune responses. J. Control. Release 242, 141-147.

Schipper, P., van der Maaden, K., Groeneveld, V., Ruigrok, M., Romeijn, S., Uleman, S., Oomens, C., Kersten, G., Jiskoot, W., Bouwstra, J., 2017. Diphtheria toxoid and Ntrimethyl chitosan layer-by-layer coated $\mathrm{pH}$-sensitive microneedles induce potent immune responses upon dermal vaccination in mice. J. Control. Release 262, 28-36.

Schipper, P., van der Maaden, K., Romeijn, S., Oomens, C., Kersten, G., Jiskoot, W., Bouwstra, J., 2016b. Determination of depth-dependent intradermal immunogenicity of adjuvanted inactivated polio vaccine delivered by microinjections via hollow microneedles. Pharm. Res. 33, 2269-2279.

Termeer, C., Benedix, F., Sleeman, J., Fieber, C., Voith, U., Ahrens, T., Miyake, K., Freudenberg, M., Galanos, C., Simon, J.C., 2002. Oligosaccharides of Hyaluronan activate dendritic cells via toll-like receptor 4. J. Exp. Med. 195, 99-111.

Termeer, C.C., Hennies, J., Voith, U., Ahrens, T., Weiss, J.M., Prehm, P., Simon, J.C., 2000. Oligosaccharides of hyaluronan are potent activators of dendritic cells. J. Immunol. 165, 1863-1870.

van der Maaden, K., Jiskoot, W., Bouwstra, J., 2012. Microneedle technologies for (trans) dermal drug and vaccine delivery. J. Control. Release 161, 645-655.

van der Maaden, K., Sekerdag, E., Jiskoot, W., Bouwstra, J., 2014a. Impact-insertion applicator improves reliability of skin penetration by solid microneedle arrays. AAPS J. $16,681-684$.

van der Maaden, K., Trietsch, S.J., Kraan, H., Varypataki, E.M., Romeijn, S., Zwier, R., van der Linden, H.J., Kersten, G., Hankemeier, T., Jiskoot, W., Bouwstra, J., 2014b. Novel hollow microneedle technology for depth-controlled microinjection-mediated dermal vaccination: a study with polio vaccine in rats. Pharm. Res. 31, 1846-1854.

Verhoeff, A.A., Brand, R.P., Lekkerkerker, H.N.W., 2011. Tuning the birefringence of the nematic phase in suspensions of colloidal gibbsite platelets. Mol. Phys. 109, 1363-1371.

Vogelbruch, M., Nuss, B., Korner, M., Kapp, A., Kiehl, P., Bohm, W., 2000. Aluminiuminduced granulomas after inaccurate intradermal hyposensitization injections of aluminium-adsorbed depot preparations. Allergy 55, 883-887.

Wierenga, A.M., Lenstra, T.A.J., Philipse, A.P., 1998. Aqueous dispersions of colloidal gibbsite platelets: synthesis, characterisation and intrinsic viscosity measurements. Colloids Surf. a-Physicochem. Eng. Aspects 134, 359-371.

Zhu, Z., Ye, X., Ku, Z., Liu, Q., Shen, C., Luo, H., Luan, H., Zhang, C., Tian, S., Lim, C., Huang, Z., Wang, H., 2016. Transcutaneous immunization via rapidly dissolvable microneedles protects against hand-foot-and-mouth disease caused by enterovirus 71 . J. Control. Release 243, 291-302. 